\title{
Empirical Research on Stock Type Fund Quantitative Investment in Chinese Market Based on Enhanced Markowitz Model
}

\author{
Liangyu Min ${ }^{1}$ \\ Capital University of Economics and Business, BeiJing 100070, China \\ ${ }^{a}$ email,
}

Keywords: Stock Type Fund; Quantitative Investment; Markowitz Model

\begin{abstract}
To construct a portfolio under stock type fund, This paper take the idea of quantitative investment and Markowitz model, but some limitations in current policies urge us revise the traditional Markowitz model. So we develop a noval Markowitz model based on the old one to conform these conditions and take a series of empirical analysis to prove that the noval model is valuable.
\end{abstract}

\section{Introduction}

Quantitative investment is a new concept which refers totake the principle of mathematical statistics and use powerful computing ability to analyze and compare the data of stocks. Then select optimized portfolio quantitative model. Markowitz(1952) put up a classical mean-variance model, this model is widely used and developed into a basic model in quantitative investment, However, Markowitz model did not take weight limit of invest into account. The default makes Markowitz model hardly play a significant role in the construction of a stock type funds. The paper aim to enhanced classical Markowitz model and applied the new model into stock type funds. In research, $\mathrm{R}$ software is a main tool.

\section{Classic Markowitz Model}

According to the rational person hypothesis, each investor is in the pursuit of the maximization of individual utility, and the decision-making process could be simplified as follows:

$$
\begin{aligned}
& \min _{\omega_{i}} \sigma^{2}\left(R_{p}\right)=\sum_{i=1}^{N} \omega_{i}^{2} \sigma^{2}\left(R_{i}\right)+\sum_{i \neq j} \omega_{i} \omega_{j}\left(R_{i} R_{j}\right) \\
& s . t \rightarrow \bar{R}_{p}=\sum_{i=1}^{N} \omega_{i} E\left(R_{i}\right) \\
& \sum_{i=1}^{N} \omega_{i}=1
\end{aligned}
$$

Use Lagrange equation solve this model, the entire mathematical model is Programming Quadratic, under the given weights of asset, we can get the solution through first order condition.

$$
\left[\begin{array}{ccc}
\sum & 0 & \bar{R} \\
e^{\prime} & 0 & 0 \\
\bar{R} & 0 & 0
\end{array}\right]\left[\begin{array}{c}
\omega^{*} \\
\lambda_{1} \\
\lambda_{2}
\end{array}\right]=\left[\begin{array}{c}
0 \\
1 \\
\bar{R}_{p}
\end{array}\right]
$$

\section{Enhanced Markowitz Model}

Withthe new rule of stock type funds take effect on 2015-08, the stock fund positions in portfolio cannot be less than $80 \%$, but generally cannot be more than $95 \%$. These provisions make classical 
Markowitz model and some related invest strategies no longer applicable. For solving this problem in Chinese market, weight coefficient is introduced and enhanced Markowitz model is constructed, the first order matrix as follows:

$$
\left[\begin{array}{ccc}
\sum & 0 & \bar{R} \\
e^{\prime} & 0 & 0 \\
\bar{R} & 0 & 0
\end{array}\right]\left[\begin{array}{c}
\omega^{*} \\
\lambda_{1} \\
\lambda_{2}
\end{array}\right]=\left[\begin{array}{c}
0 \\
\text { weight } \\
\bar{R}_{p}
\end{array}\right]
$$

The following empirical research is based on the enhanced Markowitz model.

\section{Empirical Research}

Selection of Stock Portfolio. Assume that some constructed stock type funds in A-share market, the portfolio of stocks in this funds are selected by Chinese Sinopec(600028), Dshi Intelligent(002421), Jieshun Tech.(002609), Bank of China(601988), time interval is 2015-07-06 to 2016-06-14. If a suspension happened, we removed these day revenues from stock pool. After stock data processing in $\mathrm{R}$, we could get data as follows:

\begin{tabular}{|c|c|c|c|c|}
\hline & 00028 & 002421 & 09 & $\mathbf{8}$ \\
\hline 2015-01-06 & 0.019607843 & .0064011380 & 0.0532934132 & 0.031674208 \\
\hline $2015-01-07$ & 0.025714286 & 0.0487632509 & 0.0011370097 & -0.004385965 \\
\hline $2015-05-05$ & -0.029411765 & 0.1000673854 & 0.0061199510 & -0.049689441 \\
\hline $2015-05-06$ & -0.021464646 & 0.1001531394 & 0.0301094891 & -0.017429194 \\
\hline $2015-05-07$ & -0.033548387 & .0999443207 & 0776498 & -0.017738359 \\
\hline $2015-05-08$ & 0.0 & 6900 & .0662 & 3544018 \\
\hline $2015-$ & 0.019788918 & 1000920387 & 0.0969441517 & 0.015590200 \\
\hline $2015-05-12$ & 0.003880983 & -0.5009412257 & -0.0403458213 & 0.004385965 \\
\hline $2015-05-13$ & -0.028350515 & 0.1001676446 & 0.0735735736 & -0.004366812 \\
\hline $2015-05-14$ & 0.009283820 & 0.1001904762 & 0.0531468531 & -0.002192982 \\
\hline $2015-05-15$ & -0.039421813 & 0.1000692521 & 0.0119521912 & -0.024175824 \\
\hline $2015-05-18$ & -0.025991792 & 0.0034623859 & 0.0999562555 & -0.024774775 \\
\hline$m-\infty+\cdots$ & $-2-20-20-1$ \& & 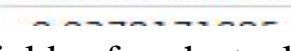 & & \\
\hline
\end{tabular}

Fig1. Yields of each stock equity portfolio

Then we would calculate the cumulative yield of each stock in our portfolio within $\mathrm{R}$ software. We could get each stock's image of daily returns and cumulative return rate as follows:

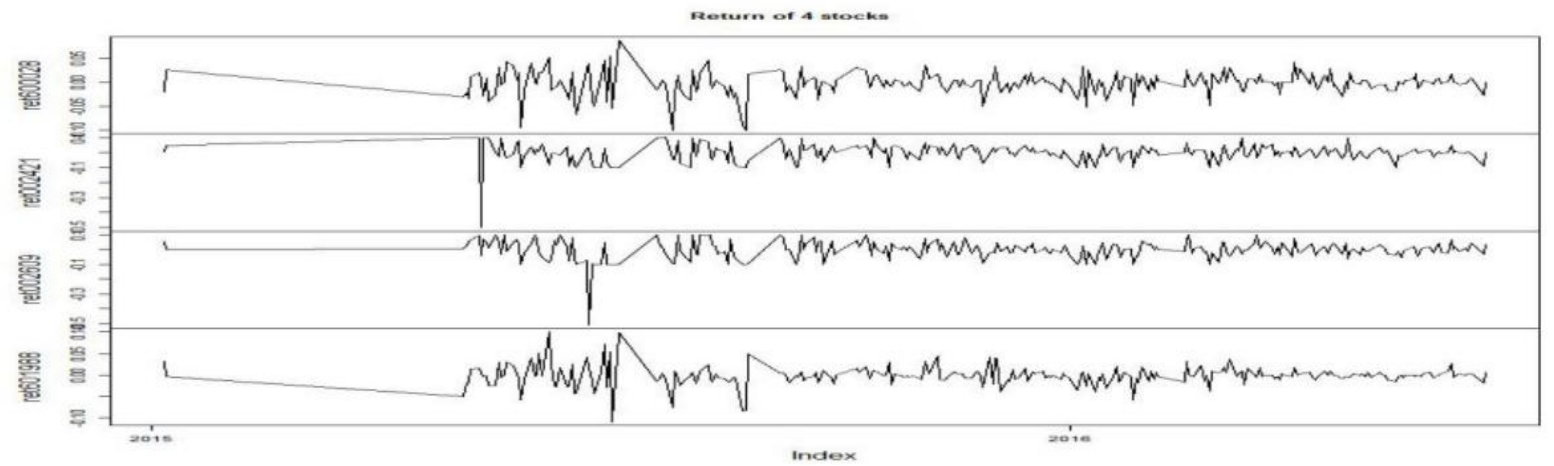

Fig2. Daily return rate image 


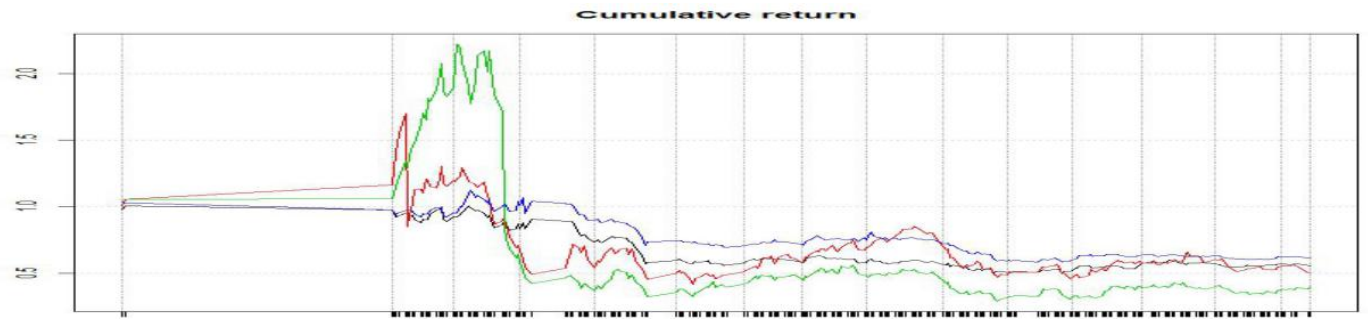

Fig3.cumulative return rate

Detection for Stock's correlation. In order to distribute non-system risk in our stock portfolio, we should detect the correlation between the stock portfolio, if selected stocks show strong correlation, it is assumed that our stock portfolio lacking in ability for distributing non-system risk. In our detection with $\mathrm{R}$, we could get the correlation coefficients matrix as follows:

\begin{tabular}{|r|r|r|r|r|}
\hline & ret600028 & ret002421 & ret002609 & ret601988 \\
\hline ret600028 & 1.0000000 & 0.3213027 & 0.3185278 & 0.8130420 \\
\hline ret002421 & 0.3213027 & 1.0000000 & 0.5053945 & 0.1717133 \\
\hline ret002609 & 0.3185278 & 0.5053945 & 1.0000000 & 0.1878448 \\
\hline ret601988 & 0.8130420 & 0.1717133 & 0.1878448 & 1.0000000 \\
\hline
\end{tabular}

Fig4.correlation coefficients matrix

It is easy to found that positive correlation between selected stocks. For wise investors, they should solve these matrix to get asset allocated ration for minimize risk in portfolio. Considering stock type funds is being researched, our ration is limited by some rules for investing, and the ration for allocated asset is between $80 \%$ and $95 \%$. Based on analysis above, we would take enhanced Markowitz model in effect. Here we set the value of weight $90 \%$, which means investing $90 \%$ asset on stock portfolio, and $10 \%$ on some fixed-income products, such as bonds(the default risk is zero).

Take Enhanced Markowitz Model. Programming in R software, we design risk function as follows, weight would take into function as a parameter named 'myweight'.

min_var_func $<$-function(asset,goal_ret,myweight)\{\#enhanced Markowitz Model

n<-ncol(asset);cov<-cov(asset);cmean<-apply(asset,MARGIN=2,FUN=mean)

La<-cbind(cov,rep(1,n),cmean);Lb<-rbind(c(rep(1,n),0,0),c(cmean,0,0))

L<-rbind(La,Lb);b<-c(rep(0,n),myweight,goal_ret)

result<-solve(L,b);weight<-result[1:n]

ret_mean<-cmean\%*\%weight;ret_var<-weight\%*\%cov\%*\%weight

return (c(ret_mean,ret_var,weight))

\}

Calculate the effective frontier of stock portfolio. In the customized risk function, we set the parameter myweight value as 0.9 , then draw the effective frontier image as follows:

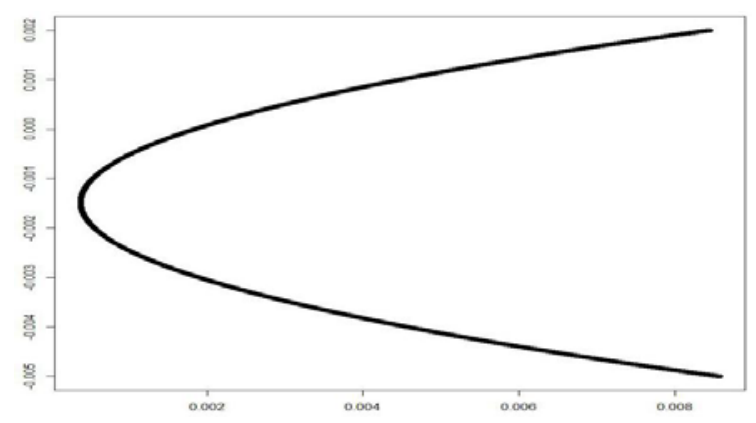

Fig5.effective frontier 
From curve above, weak performance showed on Chinese market in time interval. It is widely known that Chinese A-share Market experienced three continuous rounds stock crash between second half in 2015 and 2016.June. At the same period, Chinese economics is not ideal, the GDP index is swagging between $6 \%$ and $6.5 \%$. Based on macroeconomic conditions, we take pessimistic attitude for Chinese A-share market. For minimizing risk, our target return rate is -0.0018 . Using the enhanced Markowitz model to calculate the ratio of allocated asset.

Comparison between Training Set and Test Set. For proving the validity of enhanced Markowitz model, we take model parameters from training set and random set parameters into test set. In the training set,we set May 52015 and April 312016 as time interval. In the test set, time interval is May 1,2016 to June 14,2016. In the training set, we get asset allocated ration as follows:

\begin{tabular}{|l|l|l|l|}
\hline ret600028 & ret002421 & ret002609 & ret601988 \\
\hline 0.69164439 & -0.05672968 & 0.03534724 & 0.22973805 \\
\hline
\end{tabular}

Some ratio is negative because of the existence of short selling mechanism. Considering short selling mechanism, random parameters my be negative at the same time. We could use $\mathrm{R}$ codes to get random parameters:

$\mathrm{N}<-29$;random_wt<-t(sapply(1:N,FUN=function(i) \{\#Model of random parameters wt $<$-runif(4,min=-0.1,max=0.9);wt<-0.9*wt/sum(wt);return(wt) $\}$ ))

According to the results above, the image of cumulative return of the training model and random parameters model are as follows:

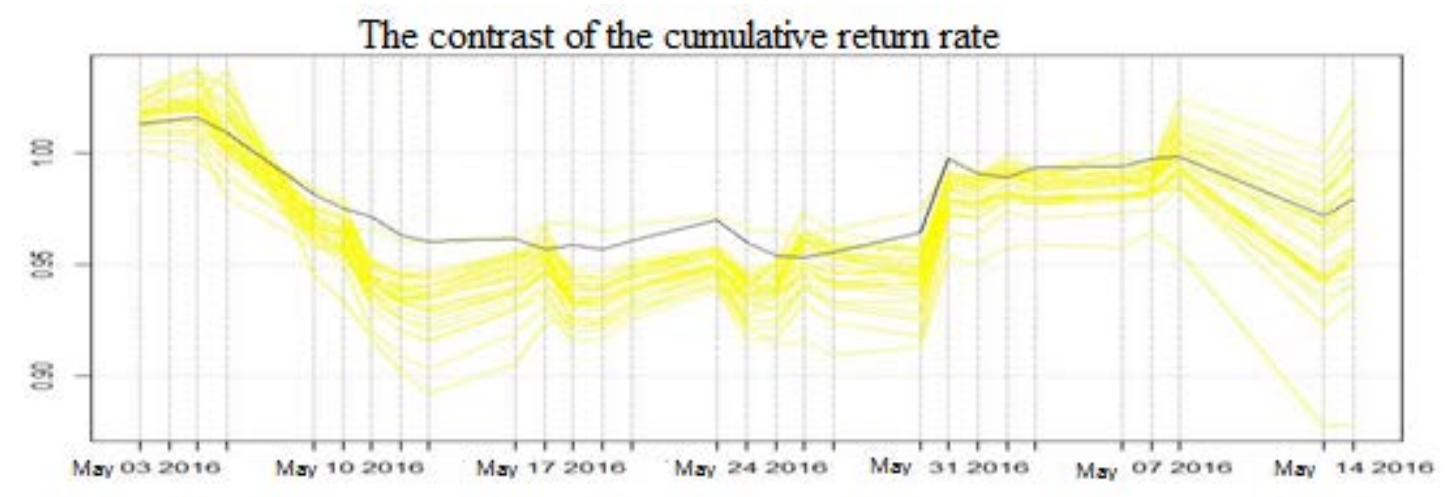

Fig6.Comparison between different parameters

Results Analysis. From the figure, Higher cumulative returns(black-line) gained in training set compared with stochastic simulation model(yellow-line). It is reasonable to think that ratios from training set is effective with certain value for investing strategies.

\section{References}

[1] Amihud Y.IIIiquidity and Stock Return;Cross-Section and Time-Series Effect[J].Journal of Financial Markets,2002,(5).

[2] Kaufman P J.New Trading System and Methods[M].John Wiley\&Sons Inc,2005.

[3] Whistler M.Trading Pairs : capturing profits and hedging risk with statistical arbitrage strategies[M].John Wiley \& Sons, 2004.

[4] Vidyamurthy, G. Pairs Trading: Quantitative Methods and Analysis[M].John Wiley \& Sons, 2004.

[5] Eillot Robert J, Van Der Hoek, John, Macolm William P.Pairs trading[J].Quantitative Finance, 2005, 5(3). 
[6] Engle, R.and Granger, C. Cointegration and Error Correction: Representation,Estimation and Testing[J].Econometrica, Vol.55, No.2, 1987.

[7] Zhao Yin, Chang Tan. Model and Analysis of Financial Investment Risk monetary quantification prediction[J]. Journal of Central University of Finance and Economics 2013, (7)

[8] Fu Yongjian, etc. Based on futures portfolio quantization ideas and Black-Litterman model[J]. Chinese Academy of Sciences University 2014, (7)

[9] Yu Liwei, Ningling stock market quantitative investment strategies and empirical test[J]. Statistics and Decision 2016, (6)

[10] Cai Lirui. Quantitative investment in R language as a tool[M]. Electronic Industry Press 